\title{
High resolution computed tomographic findings in pulmonary tuberculosis
}

\author{
O N Hatipoğlu, E Osma, M Manisali, E S Uçan, P Balci, A Akkoçlu, O Akpinar, \\ C Karlikaya, C Yüksel
}

\begin{abstract}
Background - Although chest radiographs usually provide adequate information for the diagnosis of active pulmonary tuberculosis, minimal exudative tuberculosis can be overlooked on standard chest radiographs. The aim of the present study was to assess the findings of active pulmonary tuberculosis on high resolution computed tomographic (HRCT) scans, and to evaluate their possible use in determining disease activity.
\end{abstract}

Methods - Thirty two patients with newly diagnosed active pulmonary tuberculosis and 34 patients with inactive pulmonary tuberculosis were examined. The diagnosis of active pulmonary tuberculosis was based on positive acid fast bacilli in sputum and bronchial washing smears or cultures and/or changes on serial radiographs obtained during treatment.

Results - With HRCT scanning centrilobular lesions $(n=29)$, "tree-in-bud" appearance $(n=23)$, and macronodules $5-8 \mathrm{~mm}$ in diameter $(n=22)$ were most commonly seen in cases of active pulmonary tuberculosis. HRCT scans showed fibrotic lesions $(n=34)$, distortion of bronchovascular structures $(n=32)$, emphysema $(n=28)$, and bronchiectasis $(n=$ 24 ) in patients with inactive tuberculosis. Conclusions - Centrilobular densities in and around the small airways and "treein-bud" appearances were the most characteristic CT features of disease activity. HRCT scanning clearly differentiated old fibrotic lesions from new active lesions and demonstrated early bronchogenic spread. These findings may be of value in decisions on treatment.

(Thorax 1996;51:397-402)

Keywords: tuberculosis, imaging, high resolution computed tomography.

The most common form of pulmonary tuberculosis in adults is postprimary disease or reinfection. Although chest radiographs are extremely good for the diagnosis of active pulmonary tuberculosis, minimal exudative tuberculosis can be overlooked on standard chest radiography. ${ }^{1-3}$ Computed tomography (CT) is superior to conventional radiography in detecting activity and CT features of pulmonary tuberculosis have been described. ${ }^{4-6}$ In recent years, because of high resolution power and minimal partial volume effect, high resolution computed tomography (HRCT) has been found to be superior to conventional chest radiography and standard CT in the localisation of disease in the pulmonary lobule and in the evaluation of pulmonary parenchymal disease. Using these advantages of HRCT, we have studied patients with active and inactive pulmonary tuberculosis to assess the contribution of HRCT to the evaluation of tuberculosis.

\section{Methods}

PATIENTS

Thirty two patients with active pulmonary tuberculosis and 36 patients with inactive disease were studied prospectively at our department from February 1993 to March 1994 using HRCT scanning. Two cases with inactive tuberculosis whose chest radiographs and HRCT images showed findings consistent with activity were excluded as these new lesions were due to non-tuberculous infection in old tuberculosis lesions. The group with active tuberculosis included 23 men of mean age 44 years (range 19-80). The diagnosis of active pulmonary tuberculosis was based on (1) detection of acid fast bacilli in sputum smears $(\mathrm{n}=17,53 \%)$; (2) detection of acid fast bacilli in cultures of sputum $(n=21,65 \%)$ or bronchial washings $(n=3)$; and (3) radiographic and clinical improvement after administration of two or more antituberculous drugs for patients whose clinical and radiographic findings suggested a diagnosis of pulmonary tuberculosis but smear and culture results were negative $(n=8,25 \%)$. In addition, pleural biopsy confirmed the diagnosis in two patients in whom sputum smears were also positive for acid fast bacilli. Bronchial washings were obtained from 15 of the patients with active disease who could not expectorate sputum $(\mathbf{n}=2)$ or whose sputum smears were negative for acid fast bacilli $(n=13)$. Fourteen patients had a history of previous antituberculosis therapy and, for the remaining 18 , it was the initial diagnosis.

The inactive group included 25 men of mean age 58 years (range 24-78). The diagnosis of inactive pulmonary tuberculosis was established on the basis of the following criteria: (1) findings of residual fibrotic changes on chest radiography; (2) absence of radiological progression comparing chest radiographs at recruitment with radiographs obtained six months previously or at follow up; and (3) absence of acid fast bacilli in sputum or bronchial washings on smear or culture. Bronchial washings were obtained from 12 patients who could not expectorate sputum and from two who had 
radiographic evidence of active pulmonary tuberculosis but had negative smear and culture for Mycobacterium tuberculosis. These two patients were excluded from the study (one had a positive culture of Staphylococcus aureus and the other a positive culture of Klebsiella pneumoniae). Both showed clinical and radiographic improvement after administration of appropriate antibiotics. Serial follow up radiographs were taken monthly in the inactive tuberculosis group for 3-6 months. Follow up radiographs showed no progression.

\section{IMAGING}

\section{HRCT}

Patients with active or suspected reactivation of tuberculosis on conventional chest radiographs or suspicious clinical findings underwent examination with HRCT within seven days of diagnosis; in the inactive tuberculosis group HRCT was undertaken within 3-32 days.

HRCT scans were obtained with a 9800 (GE Medical Systems, Milwaukee, USA) CT scanner. Scanning was performed with $1.5 \mathrm{~mm}$ collimation, $120 \mathrm{kvP}-170 \mathrm{~mA}$, two second scan time, $512 \times 512$ matrix, and a bone algorithm at $10 \mathrm{~mm}$ intervals from the lung apices to the bases. Intravenous contrast medium was not administered. Images were obtained with a $35-40 \mathrm{~cm}$ field of view; some were targeted retrospectively with a $20 \mathrm{~cm}$ field of view.

The terms used to interpret HRCT findings in the active cases were:

(1) Centrilobular nodule or linear structures: well defined lesions 1-4 mm thick, separated by more than $2 \mathrm{~mm}$ from the pleural surface or interlobular septa.

(2) "Tree-in-bud" appearance: a branching linear structure with more than one contiguous branching site.

(3) Macronodule: a nodule $5-8 \mathrm{~mm}$ in diameter.

In addition, consolidation (lobular, subsegmental, segmental, lobar), bronchial wall thickening, cavitation (single, multiple), emphysema, bronchovascular distortion, fibrotic changes, pleural thickening, bronchiectasis, lymphadenopathy, parenchymal calcification, pleural effusion, miliary nodules, and ground glass appearances were noted.

\section{Chest radiography}

In patients with active disease chest radiographs at the time of initial examination were reviewed, focusing on the presence of cavities (single or multiple), the distribution of lesions (unilaterally or bilaterally), and findings of activity (infiltration with or without cavitation).

\section{STATISTICAL ANALYSIS}

Comparisons between active and inactive disease were made using $\chi^{2}$ analysis and a value of $\mathrm{p}<0.05$ was regarded as significant.

Stepwise discriminant analysis as used in this study identifies the discriminating HRCT findings that most usefully measure the char-
HRCT findings of patients with active and inactive pulmonary tuberculosis

\begin{tabular}{lrrrr}
\hline Findings & $\begin{array}{l}\text { Active } \\
\text { tuberculosis } \\
(n=32)\end{array}$ & $\begin{array}{l}\text { Inactive } \\
\text { tuberculosis } \\
(n=34)\end{array}$ \\
\hline Centrilobular nodule and/or & 29 & $(91)$ & 0 & $(0)$ \\
$\quad$ branching linear structure* & 23 & $(71)$ & 0 & $(0)$ \\
Tree-in-bud appearance* & 22 & $(69)$ & 0 & $(0)$ \\
Macronodule* & 16 & $(50)$ & 4 & $(12)$ \\
Cavity* & 14 & $(44)$ & 0 & $(0)$ \\
Consolidation* & 14 & $(44)$ & 22 & $(65)$ \\
Bronchial wall thickening & 10 & $(34)$ & 3 & $(9)$ \\
Interlobular septal thickening & 12 & $(38)$ & 4 & $(11)$ \\
Ground glass opacity* & 18 & $(56)$ & 24 & $(71)$ \\
Bronchiectasis & 14 & $(44)$ & 28 & $(82)$ \\
Emphysema* & 20 & $(63)$ & 32 & $(94)$ \\
Bronchovascular distortion* & 21 & $(66)$ & 34 & $(100)$ \\
Fibrotic changes* & 7 & $(22)$ & 15 & $(44)$ \\
Calcified mediastinal lymph & 14 & $(44)$ & 18 & $(53)$ \\
$\quad$ node enlargement* & 20 & $(63)$ & 24 & $(71)$ \\
Parenchymal calcification & 5 & $(16)$ & 0 & $(0)$ \\
Pleural thickening or retraction & 2 & $(6)$ & 0 & $(0)$ \\
Lymphadenopathy $>10$ mm) & 1 & $(3)$ & 0 & $(0)$ \\
Pleural effusion & Miliary nodules & & & \\
\hline
\end{tabular}

Values in parentheses are percentages. $* \mathrm{p}<0 \cdot 05$.

acteristics in which the two groups are expected to differ. The objective is to maximise separation of groups by weighting and combining the discriminating variables in some linear form. A user selected criterion is applied, and the first variable included in the analysis has the largest acceptable value for the selection criterion. After the first variable is entered, the value of the criterion is re-evaluated for all variables not in the model and the variable with the largest acceptable criterion value is entered next. The variable entered first is then reevaluated to determine whether it meets the removal criterion. If it does it is removed from the model. The next step is to examine the variables not in the equation for removal. Variables are removed until nine remain that meet the removal criterion. Variable selection terminates when no more variables meet entry or removal criteria.

In this study the ability to classify cases correctly with the use of the discriminant function was measured; pooled within group correlations between discriminating variables and canonical discriminate functions were calculated by SPSS/PC + .

The study was approved by the hospital ethics committee.

\section{Results}

HRCT FEATURES OF ACTIVE DISEASE

The HRCT findings in patients with active pulmonary tuberculosis are summarised in the table. Centrilobular nodular or linear structures $(n=29,91 \%)(p<0.001)$ (figs 1 and 2$)$, "tree-in-bud" appearance ( $\mathrm{n}=23,71 \%)$ ( $p<0.001$ ) (figs 1 and 3 ), and macronodules $(n=22,69 \%)(p<0.001)$ (fig 4$)$ were the most common HRCT findings seen in active pulmonary tuberculosis.

Centrilobular nodules, branching linear structures, and/or "tree-in-bud" appearances were detected in 29 of the 32 patients with active tuberculosis (91\%). The exceptions were one patient with miliary tuberculosis, one 


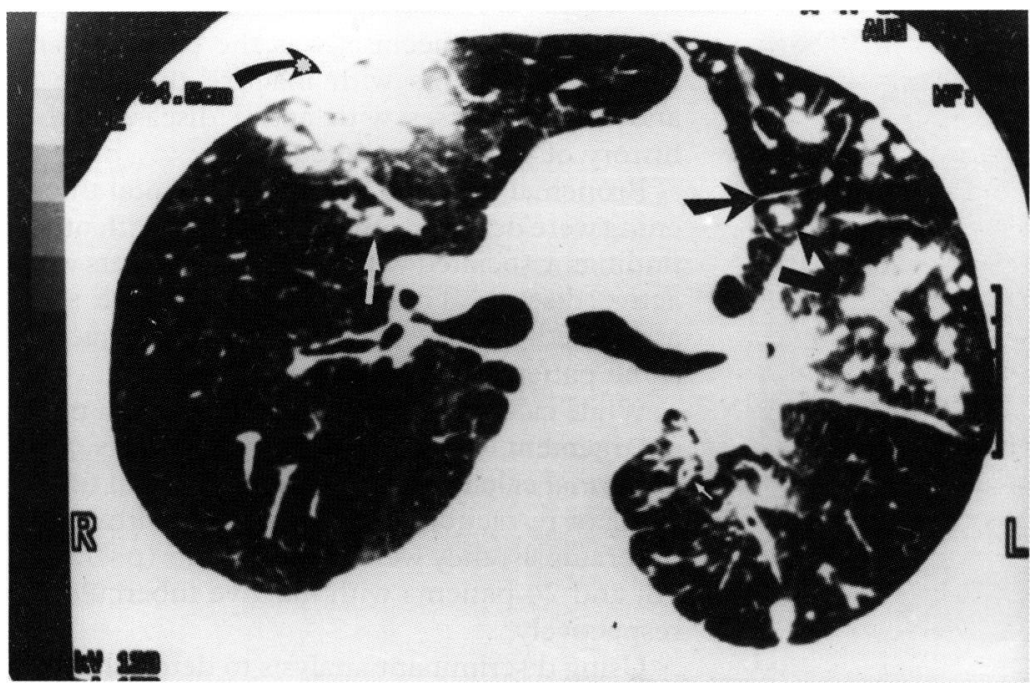

Figure 1 HRCT section at the level of the bifurcation of the trachea in a 36 year old man with sputum positive for acid fast bacilli. Centrilobular nodular densities (curved black arrow) and "tree-in-bud" appearance (white arrow) are common findings in active pulmonary tuberculosis. The appearances are due to endobronchial spreading. Coalescence of granulomas and acinar-lobular infiltrates usually cause subsegmental consolidation areas (black arrow with asterisk). Interlobular septal thickening (straight black arrow) is also a common entity observed in active tuberculosis.

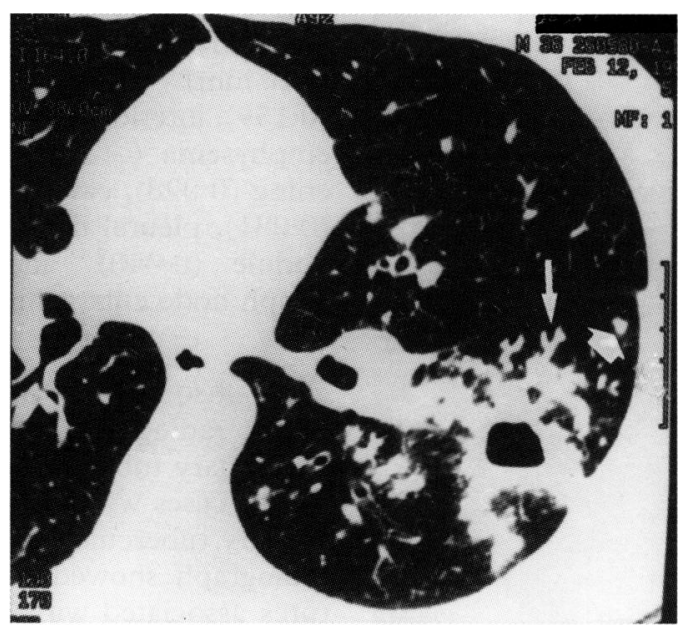

Figure 2 HRCT section at the mid lung level in a 42 year old man with sputum positive for acid fast bacilli. The long white arrow shows a centrilobular linear branching structure; centrilobular nodules (thick white arrow) and cavities are also seen.

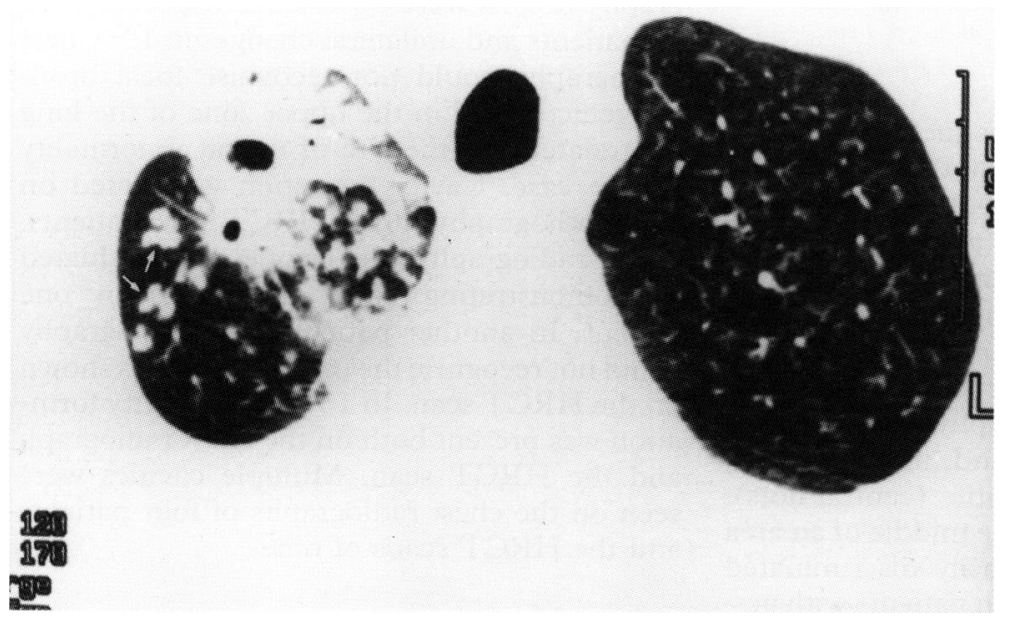

Figure 3 HRCT section at the apices of the lungs in a woman aged 51 years with sputum positive for acid fast bacilli. "Tree-in-bud" appearances (white arrows) and accompanying cavities are shown. patient with lobar consolidation, and one patient with a history of previous antituberculous therapy. Since lymphohaematogenous dissemination occurs in miliary tuberculosis, we could not detect findings of early bronchogenic spread. In the patient with lobar consolidation we thought that small nodular lesions had resulted in lobular consolidation and that these had led to segmental and finally lobar consolidation. In the third patient, although we could not observe centrilobular nodules, branching linear structures, or "tree-in-bud" appearance, a macronodule and an area of lobular consolidation were present. In these three patients the diagnosis of active pulmonary tuberculosis had been confirmed by positive acid fast bacilli on smear and/or culture.

The characteristic findings of bronchogenic spread were detected in 30 of 31 patients $(97 \%)$ with postprimary tuberculosis (all except the individual with lobar consolidation). None of these lesions was detected in patients with inactive pulmonary tuberculosis. In one of the two cases excluded, a macronodule was present and subsegmental consolidation was shown in the other case. Bronchiectasis in the upper lobes was present in both.

Pulmonary consolidation (fig 1) was seen in $14(44 \%)$ patients with active tuberculosis $(p<0.001)$ and it was not encountered in the group with inactive disease. A ground glass appearance was present in $12(38 \%)$ of the patients with active tuberculosis and was common in areas surrounding nodular lesions $(p<0.05)$. This appearance was seen, associated with fibrotic changes, in a minority of patients with inactive disease.

Cavitation was present in $16(50 \%)$ of the patients with active tuberculosis $(p<0.001)$. The cavity walls ranged from very thin and smooth to very thick and nodular. Bronchogenic spread of tuberculous material from cavities to other parts of the lung was always present in patients with active disease. Cavitation was present in four of the patients with inactive disease. Most cavity walls were thin and evidence of bronchogenic spread was absent. Five patients with active tuberculosis had non-calcified mediastinal lymphadenopathy $(>10 \mathrm{~mm})$. In most of these cases the findings of disseminated pulmonary tuberculosis were present. In patients with inactive tuberculosis no mediastinal lymphadenopathy over $10 \mathrm{~mm}$ was detected.

Pleural effusion was found in two of the patients with active tuberculosis. The effusion was associated with parenchymal infiltration and was positive for acid fast bacilli; pleural biopsy confirmed the diagnosis.

Multiple discrete miliary nodules (fig 5) were seen in an even distribution throughout the lung in one case with miliary tuberculosis. The diagnosis was confirmed by a positive culture for Mycobacterium tuberculosis.

HRCT FEATURES IN INACTIVE DISEASE

The HRCT findings in inactive pulmonary tuberculosis are summarised in the table. The 


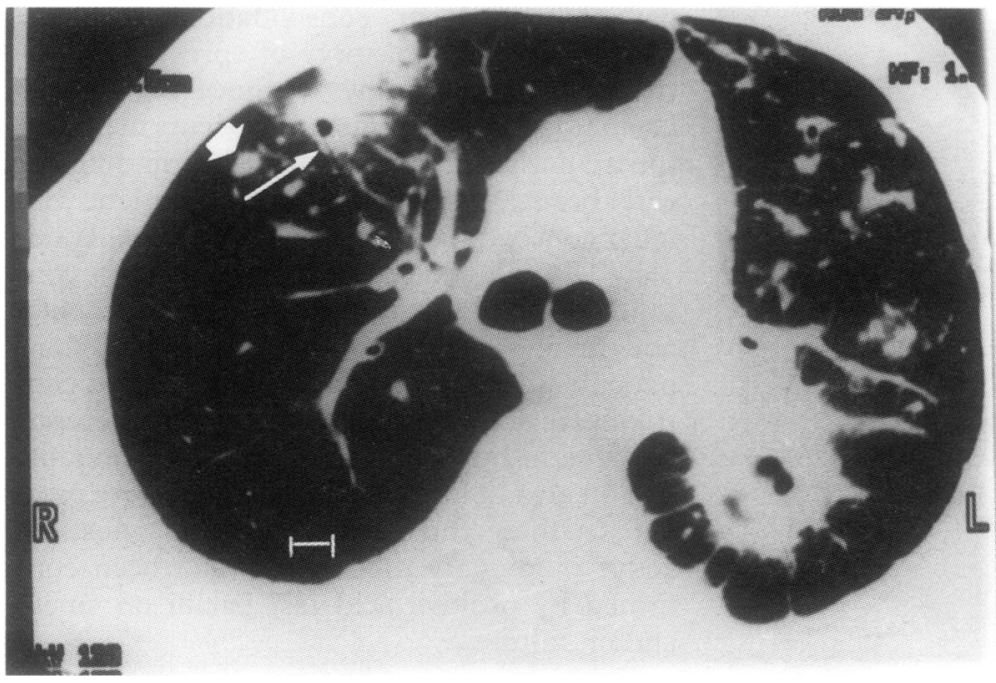

Figure 4 HRCT section at the level of the bifurcation of the trachea in a man aged 36 years with sputum positive for acid fast bacilli. Increased spatial resolution offers the opportunity of demonstrating drainage bronchioles of tuberculous cavities (long white arrow); inferior to the cavity a macronodule (thick white arrow) is seen. Bar indicates $1 \mathrm{~cm}$.
Traction bronchiectasis was the predominant feature in patients with inactive tuberculosis and in those cases with active disease and a history of previous tuberculosis.

Bronchial wall and interlobular septal thickening were detected adjacent to areas with other findings associated with activity in patients with active disease. These appearances were seen associated with the residual fibrotic changes in some patients with inactive disease.

While calcified mediastinal/hilar lymph node enlargement was present in seven patients, parenchymal calcification in 14, and pleural thickening or retraction in 20 individuals with active tuberculosis, they were present in $15(\mathrm{p}<0.05)$, 18 , and 24 patients with inactive tuberculosis, respectively.

Using discriminant analysis to determine the HRCT features which best correlated with activity we found that the variables, ordered by magnitude of correlation, were: centrilobular nodule (0.703), "tree in bud" appearance $(0.539)$, macronodule $(0.467)$, bronchovascular distortion $(-0.361)$, fibrotic changes $(-0 \cdot 290)$, parenchymal calcification $(-0.262)$, bronchiectasis $(-0.214)$, consolidation (0.199), lymphadenopathy $(>10 \mathrm{~mm}) \quad(0 \cdot 188)$, ground glass opacity $(0 \cdot 159)$, interlobular septal thickening $(0 \cdot 154)$, emphysema $(-0 \cdot 152)$, bronchial wall thickening (0.098), cavity $(0.096)$, pleural effusion $(0.081)$, pleural thickening $(-0.045)$, miliary nodule $(0.040)$, and calcified mediastinal lymph node enlargement $(-0 \cdot 017)$.

\section{COMPARISON WITH CHEST RADIOGRAPHY}

Chest radiographic findings consistent with pulmonary tuberculosis were present in 17 of the 18 cases with newly diagnosed active pulmonary tuberculosis. In one patient the chest radiograph showed minimal change but features associated with activity were recognised using HRCT. New lesions around old fibrotic lesions were easily demonstrated by chest radiography in seven of 14 cases with a history of previous antituberculous treatment and a suspicion of reactivation in the remaining seven cases. HRCT could easily distinguish new lesions from old fibrotic lesions. On chest radiography lesions were observed in both lungs in 13 patients and unilateral changes in 19 . Chest radiography could not recognise focal bronchogenic spread in the upper zone of the lung contralateral to the site of major abnormality in one case. Cavity formation was noted on chest radiography and on HRCT in 16 patients. Chest radiography was erroneously evaluated as demonstrating cavity formation in one patient. In another patient chest radiography could not recognise the cavity which was shown on the HRCT scan. In 15 patients cavity formation was present both on the chest radiograph and the HRCT scan. Multiple cavities were seen on the chest radiographs of four patients and the HRCT scans of nine.

\section{Discussion}

Postprimary or reactivation tuberculosis is the most common form of pulmonary tuberculosis of emphysema could be easily discril from centrilobular nodules in patients with active tuberculosis. Bronchiectasis was detected in $18(56 \%)$ of the patients with active disease and in $24(71 \%)$ of those with inactive disease. 


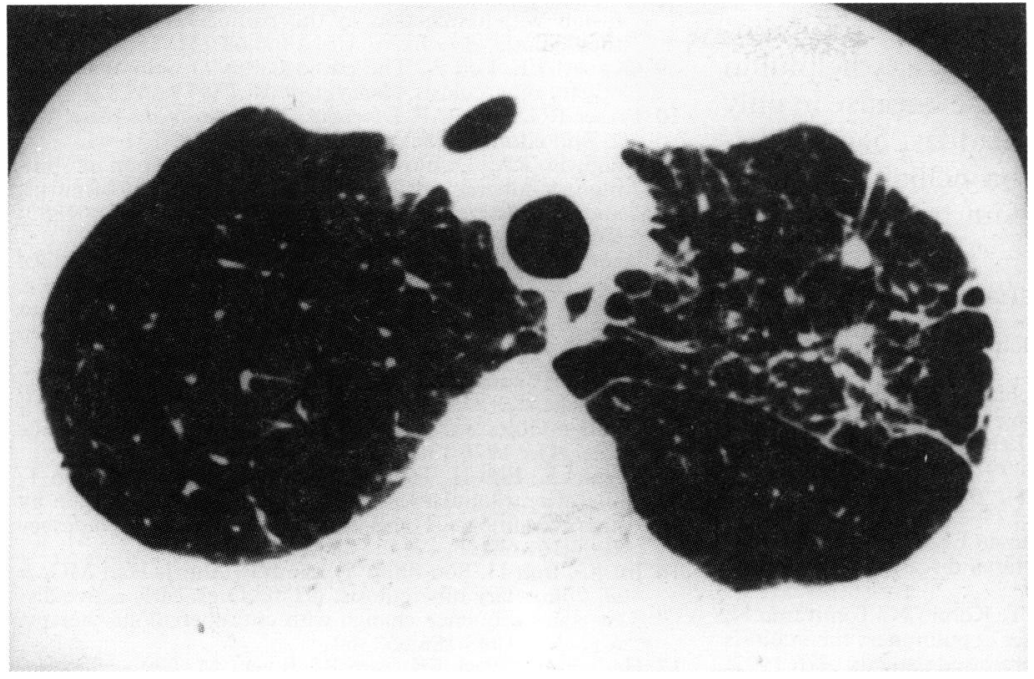

Figure 6 HRCT section at the subapical level in a patient aged 62 years with inactive tuberculosis. On the left, irregular fibrotic changes causing parenchymal distortion and traction bronchiectasis are seen. There are also emphysematous areas.

in adults. Various investigations have concluded that postprimary tuberculosis results from reactivation of a previously dormant primary infection in $90 \%$ of cases..$^{7-9}$ A few cases represent a continuation of the primary disease. ${ }^{810}$ Rarely, postprimary tuberculosis is a result of exogenous superinfection on inactive or even active original infection. The early parenchymal infiltrate of postprimary tuberculosis results from an intense inflammatory reaction in the hypersensitive host. ${ }^{11}$ This may lead to caseous necrosis which over time is apt to slough into a bronchus, leaving a cavity. ${ }^{11}$ The most commonly involved areas of the lungs in postprimary disease are the apical and posterior segments of an upper lobe. ${ }^{1-310-12}$ Bronchogenic dissemination is the most common means of spread in postprimary tuberculosis. The typical chest radiographic finding in postprimary tuberculosis is an acinar pattern - that is, multiple fluffy nodules roughly $5 \mathrm{~mm}$ in diameter. ${ }^{10}$ Such a radiographic pattern has been reported in various diseases. ${ }^{1013}$ Itoh et $a l^{14}$ showed in their necroscopic studies that the disease develops initially in respiratory bronchioles followed by surrounding focal inflammation, and that these pathological processes are reflected in the nodules seen on radiological examination of the lung. Smaller nodules evolved into larger lesions whose diameter was the same as that of the pulmonary acinus, and it was inferred that direct extension of inflammation through the pores of Kohn or aspiration of inflammatory materials into the pulmonary acinus was the mechanism involved. In addition, caseous necrosis was shown to affect the walls of the bronchioles and adjacent alveoli. Murata et $a l^{15}$ showed that these peribronchiolar intralobular nodules could be recognised as centrilobular nodules on HRCT scans and that, in tuberculosis, they were found around the respiratory bronchioles.

Im et $a l^{16}$ reported that the earliest HRCT finding of bronchogenic dissemination of tuberculosis was a centrilobular nodule or branching linear structure $2-4 \mathrm{~mm}$ in diameter. These centrilobular lesions consisted of solid caseous material within or around the terminal or respiratory bronchioles. Multiple branching linear structures of similar calibre originating from a single "stalk" (the "tree-in-bud" appearance) were commonly seen in patients with extensive bronchogenic spread. Terminal tufts of the "tree-in-bud" structure might represent lesions within the bronchioles and alveolar ducts, while the "stalk" might represent a lesion that affected the last order bronchus within the secondary lobule. In addition they showed that bronchial wall thickening occurred during bronchogenic dissemination, that interlobular septal thickening resulted from increased lymphatic clearance, and that the ground glass appearance could be present around active lesions.

In several investigations chest radiographic findings of bronchogenic spread have been reported in approximately $20 \%$ of instances. ${ }^{1718}$ With HRCT scanning we have detected features characteristic of bronchogenic spread in 30 of 31 cases $(97 \%)$ with postprimary tuberculosis (all except for one case with lobar consolidation). The results of our study are consistent with those of Im et al. ${ }^{16}$

Our study has illustrated several advantages of HRCT scanning in cases of suspected active tuberculosis. HRCT demonstrated features associated with disease activity in a newly diagnosed patient with a normal chest radiograph, and in seven patients with indeterminate radiographs and a history of previous tuberculosis. In only one of these eight patients were acid fast bacilli detected by smear examination of the sputum and/or bronchial washings. Thus, HRCT scanning enabled an early diagnosis to be made and treatment started immediately by showing characteristic features of early bronchogenic spread. HRCT scanning was also found to be superior to chest radiography in the detection of dissemination of lesions and the presence and number of cavities.

Healing of tuberculosis lesions results in the development of a fibrotic scar and calcification with loss of lung parenchyma volume. Rarely, if lesions heal without development of necrosis, no residual fibrotic changes occur. Im et al ${ }^{16}$ showed that healing of cavitatory lesions caused more cicatricial change than in other areas without cavitation and that distortion of bronchovascular structures, emphysema, fibrotic bands, or bronchiectasis tended to increase in prevalence on follow up CT scans. In our study the frequency of bronchovascular distortion, fibrotic changes, emphysema, and bronchiectasis in patients with inactive pulmonary tuberculosis was high. This high frequency might be due to the presence of residual fibrotic changes on the chest radiographs of all patients included in the study with the diagnosis of inactive pulmonary tuberculosis.

In conclusion, our data support the concept that HRCT scanning can be of value in situations where chest radiographs do not contribute to the diagnosis of active disease and cannot distinguish new lesions from old fibrotic changes. If there is clinical suspicion, HRCT can be used to show centrilobular lesions which 
are the most common findings of early bronchogenic spread. This is particularly helpful in the management of the disease because in only $53 \%$ of our patients were acid fast bacilli present in the sputum or bronchial washings. HRCT aided decisions about treatment.

1 Miller WT, MacGregor RR. Tuberculosis: Frequency of unusual radiographic findings. $A \mp R$ 1978;130:867-75.

2 Miller WT. Tuberculosis in adults. Postgrad Radiol 1981;1 147-67.

3 Woodring $\mathrm{JH}$. The radiographic manifestations of pulmonary tuberculosis. $\mathcal{F}$ Ky Med Assoc 1984;82:17-23

4 Naidich DP, McCouley DI, Leitman BS, Genesier NB, Hulnick DH. CT of pulmonary tuberculosis. In: Siegelman SS, ed. Computed tomography of the chest. New York: Churchill Livingstone, 1984:175-217.

5 Kuhlman JE, Deutsch JH, Fishman EK, Siegelman SS. CT features of thoracic mycobacterial disease. RadioGraphics 1990;10:413-31.

6 Ikezoe J, Takeuchi N, Johkoh T, Kohno N, Tomiyama N, Kozuka T, et al. CT appearance of pulmonary tuberculosis in diabetic and immunocompromised patients. AfR 1992, 159:1175-9.

7 Stead WW. Pathogenesis of the sporadic case of tuberculosis. $N$ Engl F Med 1967;277:1008-12.

8 Stead WW, Kerby GR, Schlueter DP, Jordahl CW. The clinical spectrum of primary tuberculosis in adults: con- fusion with reinfection in the pathogenesis of chronic tuberculosis. Ann Intern Med 1968;68:731-45.

9 Geppert EF, Leff A. The pathogenesis of pulmonary and Geppert EF, Leff A. The pathogenesis of pulmonary and
miliary tuberculosis. Arch Intern Med 1979;139:1381-3.

10 Fraser RG, Pare JAP. Diagnosis of diseases of the chest. Vol. 2. 2nd edn. Philadelphia: Saunders, 1978: 731-61

11 Goodwin RA, DesPrez RM. Apical localization of pulmonary tuberculosis, chronic pulmonary histoplasmosis, and progressive massive fibrosis of the lung. Chest 1983; 83:801-5.

12 Jacobson HG, Shapiro JH. Pulmonary tuberculosis. Radiol Clin North Am 1963;1:411-27.

13 Ziskind MM, Weill H, Payzant AR. The recognition and significance of acinus filling process of the lungs. Am Rev Respir Dis 1963;87:551-9.

14 Itoh $H$, Tokunaga S, Asamato H, Furata M, Funamoto Y, Kitachi $M$, et al. Radiologic-pathologic correlation of small lung nodules with special reference to peribronchial nodules. $A \Im R$ 1978;130:233-31.

15 Murata $\mathrm{K}$, Itoh $\mathrm{H}$, Todo G, Kanaoka $\mathrm{M}$, Noma S, Itoh T, et al. Centrilobular lesions of the lung: demonstration by high resolution CT and pathologic correlation. Radiology

16 Im JG, Itoh H, Soo Shim Y, Lee HL, Ahn J, Han MC, et al. Pulmonary tuberculosis: CT findings, early active disease and sequence change with antituberculous therapy. ease and sequence change

17 Hadlock FP, Park SK, Awe RJ, Rivera M. Unusual radiographic findings in adult pulmonary tuberculosis. $A f R$ 1980;134:1015-8.

18 Woodring $\mathrm{JH}$, Vandiviere HM, Fried AM, Dillon ML, Williams TD, Melvin IG. Update: the radiographic features of pulmonary tuberculosis. AFR 1986;146:497-506. 